\title{
O Fenômeno do Expatriado no Contexto Esportivo
}

\author{
Fernanda Torres Faggiani \\ Pontifícia Universidade do Rio Grande do Sul, \\ Porto Alegre, RS, Brasil. \\ Artur Strey \\ Pontifícia Universidade do Rio Grande do Sul, \\ Porto Alegre, RS, Brasil. \\ Daniel Fulginiti \\ Pontifícia Universidade do Rio Grande do Sul, \\ Porto Alegre, RS, Brasil. \\ Carolina Saraiva de Macedo Lisboa \\ Pontifícia Universidade do Rio Grande do Sul, Porto Alegre, RS, Brasil. \\ Daniele Lindern \\ Pontifícia Universidade do Rio Grande do Sul, \\ Porto Alegre, RS, Brasil. \\ Paula Fernandes Aiquel \\ Pontifícia Universidade do Rio Grande do Sul, \\ Porto Alegre, RS, Brasil. \\ Camila Sartori \\ Pontifícia Universidade do Rio Grande do Sul, \\ Porto Alegre, RS, Brasil.
}

\begin{abstract}
Resumo: $\mathrm{O}$ presente artigo teve como objetivo identificar o(s) termo(s) utilizados nos estudos para se referirao processodemudançaderegião/paísparaapráticaesportiva.Buscou-setambémcompreender os impactos estudados referentes ao processo de adaptação do indivíduo a um novo contexto para a atividade esportiva. Uma revisão sistemática nas bases PsycNET, Web of Science eSciELO foi conduzida a partir dos descritores: Expatriates AND Sport, Acculturation AND Sport, Migratory Transition AND Sport e Migrants AND Sport. Após filtro baseado em critérios de inclusão e exclusão relacionados aos objetivos da revisão, foram identificados nove artigos. Observou-se que não existe um único termo para definir o processo de transição entre culturas de atletas, identificando-se termos como: transição migratória, migração, aculturação. O interesse dos estudos está em entender como ocorre o processo de adaptação de indivíduos em uma nova cultura. Foi possível entender que, embora motivados a uma projeção na carreira esportiva, os atletas enfrentam desafios como: adaptação ao novo estilo de treinamento, a distância da família e de amigos e a dificuldade em comunicar-se efetivamente com as pessoas da cultura local. Os atletas enfrentam situações de isolamento e dificuldades de estabelecer relações o que interfere na saúde mental e performance esportiva.
\end{abstract}

Palavras-chave: Expatriado, Aculturação, Migração, Esporte.

\section{The Phenomenon of Expatriates in the Sports Context}

\begin{abstract}
This article aimed to identify the terms used in studies to refer to the process of changing country/region for reasons related to sports practice. It also aimed to understand the studied impacts concerning the process of adaptation of the individual to a new context due to sports practice. A systematic review on the PsycNET, Web of Science and Scielo databases was conducted from the descriptors: Expatriates AND Sport, Acculturation AND Sport, Migratory Transition AND Sport and Migrants AND Sport. After applying a filter based on criteria of inclusion and exclusion related to the objectives of the review, we identified nine articles. It was observed that there is not a single term to define the transition of athletes between cultures, identifying terms such as: migratory transition, migration, acculturation. The interest of the studies is to understand how the adaptation process of individuals into a new culture occurs. It was possible to understand that although athletes are motivated by reaching projection in their sports careers, they face challenges such as: adaptation to a new training style; distance from family and childhood friends; and difficulty in effectively communicating with people of the local culture. Athletes undergo situations of isolation and difficulties for establishing relationships, which interferes with their mental health and sports performance.
\end{abstract}

Keywords: Expatriate, Acculturation, Migration, Sport. 


\title{
El Fenómeno de los Expatriados en el Contexto Deportivo
}

\begin{abstract}
Resumen:Este artículo pretende identificar el(los) término(s) utilizado(s) en los estudios para referirse al proceso de cambio país/región para la práctica deportiva. También se buscó comprender los impactos estudiados sobre el proceso de adaptación del individuo a un nuevo contexto para la actividad deportiva. Se realizó una revisión sistemática en las bases de PsycNET, Web of Science y Scielo de los descriptores: Expatriates AND Sport, Acculturation AND Sport, Migratory Transition AND Sport e Migrants AND Sport. Después de aplicar un filtro basado en criterios de inclusión y exclusión relacionados con los objetivos de la revisión, nueve artículos fueron identificados. Se observó que no existe un término único para definir el proceso de transición entre las culturas de los atletas, identificándose términos tales como: transición migratoria, migración, aculturación. El interés de los estudios es entender cómo ocurre el proceso de adaptación de los individuos a una nueva cultura. Es posible entender que aunque motivados por alcanzar proyección en su carrera deportiva, los atletas enfrentan retos como: adaptación al nuevo estilo de formación; distancia de familia y amigos; y dificultad para comunicarse efectivamente con personas de la cultura local. Los atletas se enfrentan a situaciones de aislamiento y a dificultades para establecer relaciones, lo que interfiere con la salud mental y el rendimiento deportivo. Palabras clave: Expatriados, Aculturación, Migración, Deport.
\end{abstract}

\section{Introdução}

Para constituir e consolidar a carreira esportiva, os atletas em geral necessitam transitar por diferentes clubes, em diferentes cidades e estados ou até mesmo em um distinto país. Nesta transição, podem enfrentar fatores de risco como: isolamento, competitividade, saudade dos familiares, assédio da mídia e dos fãs e perda de privacidade (Richardson, Litterwood, Nesti, \& Benstead, 2012; Weedon, 2011). Com a globalização do esporte, a mobilidade está ainda mais enfatizada e os atletas acabam deslocando-se para diferentes regiões do país e do exterior por diferentes razões, tais como: recompensa financeira, oportunidade de ascensão no esporte, experiência em outras culturas, entre outros (Richardson et al., 2012).

A transição de atletas em diferentes culturas para a prática de esporte é um tema emergente na literatura esportiva, tanto nacional como internacional, e denomina-se, na literatura nacional, como expatriado. O termo expatriado é encontrado em publicações nacionais, sobretudo em estudos que investigam o deslocamento de trabalhadores para outros países (Carpes, Scherer, Diniz, \& Beuron, 2011; Kubo, \& Braga, 2013; Pereira, Pimentel, \& Kato, 2005; Reis, 2015; Rosal, 2015).

O interesse em estudar este fenômeno está relacionado à forma como os atletas irão lidar com a nova realidade e adaptar-se a esta, podendo influen- ciar e comprometer a saúde mental e o desempenho esportivo (Agergaard, 2008; Agergaard, \& Ryba, 2014; Baines, 2013; Blodgett et al., 2014; Brandão, Magnani, Tega, \& Medina, 2013; Evans, \& Stead, 2012; Rial, 2008; Richardson et al., 2012; Ryska, 2001; Schinke, McGannon, Battochio, \&Wells, 2013; Tibbert, Andersen, \& Morris, 2015; Weedon, 2011). Observa-se que os atletas que deslocam-se para atuar em outra cidade ou país, em sua grande maioria, chegam nos novos contextos, muitas informações sobre o lugar que irão residir. Estes indivíduos podem tornarem-se os "sem pátria", vivendo em conflito em relação a sua identidade cultural (Brandão et al., 2013).

O atleta, ao mudar de cidade e mudar de clube, pode enfrentar sentimentos de medo e inseguraça ao se deparar com suas fraquezas, assim como pode se surpreender com sua capacidade de superação. $\mathrm{O}$ atleta precisa estar em equilíbrio afetivo e psicossocial no ambiente em que vive para poder manter e melhorar seu desempenho esportivo em campo (Richardson et al., 2012; Tibbert et al., 2015).

A experiência de adaptação a um novo ambiente, as possíveis dificuldades no novo estilo de vida e o desafio ao integrar um diferente estilo de vida pessoal associado a um novo ambiente profissional ilustram a complexidade desse processo de mudança. Pode-se observar conflito e sofrimento psíquico nos atletas, quando estes tentam manter o 
bom desempenho e, ainda, sentem-se pressionados a fazer jus ao investimento feito em si pelo novo clube (Richardson et al., 2012).

A mudança e a adaptação do atleta a uma nova cultura e a relação deste processo com o fracasso no esporte e o sofrimento psíquico destes indivíduos merecem atenção. Nesse sentido, o presente artigo realizou uma revisão sistemática para investigar qual o termo que vem sem sendo utilizado nos estudos para se referir ao processo de mudança de região/ país para a prática esportiva. Além disso, é também pergunta de pesquisa desta revisão compreender os impactos/efeitos que vêm sendo estudados nas pesquisas referentes à transição do atleta para jogar em um novo contexto esportivo.

\section{Método}

$\mathrm{O}$ presente artigo consistiu em um estudo de revisão sistemática da literatura, baseado no método Prefered Reporting Items for Systematic Reviews and Meta-Analyses PRISMA (Moher, Liberati, Tetzlaff, \& Altman, 2009; Urrútia, \& Bonfill, 2010), conduzido por quatro pesquisadores, de forma independente e cega. A busca de artigos foi realizada no dia 13 de abril de 2015 nas seguintes bases de dados eletrônicas, separadamente: American Psychological Association (APA - PsycNET), Web of Science, Scientific Eletronic Library (SciELO). Estas bases de dados foram escolhidas por serem consideradas referência para publicações nacionais e internacionais. Os descritores utilizados para a busca de artigos foram definidos a partir dos termos indexados no vocabulário estruturado DeCS (Descritores em Ciências da Saúde), assim como termos encontrados nas palavras-chave de artigos sobre este assunto: Expatriates AND Sport, Acculturation AND Sport, Migratory Transition AND Sport e Migrants AND Sport. As buscas iniciais resultaram em 303 artigos, segundo indicado na Figura.

Como critérios de inclusão dos artigos : 1) tratar-se de artigo empírico, 2) estudos que abordem o processo de expatriado, aculturação, migração ou transição migratória no esporte. Foram excluídos os artigos: 1) artigos repetidos nas bases de dados; 2) trabalhos que não fossem artigos científicos; 3) produções publicadas em idiomas que não inglês, espanhol ou português; 4) produções que não apresentavam referências bibliográficas; 5) artigos que não apresentavam resumo; 6) que não eram de acesso livre; 7) artigos que mencionavam apenas um dos temas (ou só processo de mudança ou só o esporte). Foram primeiramente excluídos 81 artigos repetidos. A Figura 1 mostra o processo de busca e seleção de artigos por meio de um fluxograma.

Durante o processo de análise e extração de dados foram localizados estudos que abordavam a transição de animais e a adaptação de comunidades a culturas diferentes. E, por fim, após a aplicação dos critérios de inclusão e exclusão pelos juízes, identificaram-se 37 artigos que respondiam às perguntas de pesquisa do estudo (Figura 1).

Nas situações em que a leitura dos títulos e resumos não foram suficiente para a definição sobre a inclusão ou não do artigo na revisão sistemática, estes foram mantidos e submetidos à análise na etapa seguinte do estudo: leitura dos artigos na íntegra. Neste momento, além dos critérios de inclusão e exclusão já apresentados, foi estabelecido um novo critério de inclusão: que os estudos abordem o processo de expatriado, aculturação, migração, transição migratória na perspectiva do atleta especificamente para a prática de uma modalidade esportiva. Assim, foram novamente aplicados os critérios estabelecidos nessa pesquisa e novas exclusões foram verificadas, totalizando nove artigos. Salienta-se que foram incluídos somente os estudos que abordavam o processo a partir da perspectiva do atleta.

\section{Resultados e discussão}

A presente revisão sistemática localizou um pequeno volume de trabalhos científicos e empíricos recentemente produzidos sobre expatriado. Conforme indicado na Figura, tanto o resultado das procuras iniciais $(\mathrm{N}=303$ artigos identificados), quanto os achados obtidos no final da estratégia de busca ( $\mathrm{N}$ = nove artigos encontrados) sugerem que trata-se de uma temática emergente, porém, ainda, que tem recebido pouca atenção e investimento em pesquisas em Psicologia esportiva, uma vez que aplicando um filtro para apenas artigos empíricos, o número de produções diminui consideravelmente. Entretanto, constata-se que o expatriado está sendo investigado em países de grande repercussão em estudos da Psicologia do esporte e que representam grandes potências esportivas. Foram localizadas produções científicas na Europa (Inglaterra $=4$; Dinamarca =2), na América do Norte (Canadá=2) e Oceania 

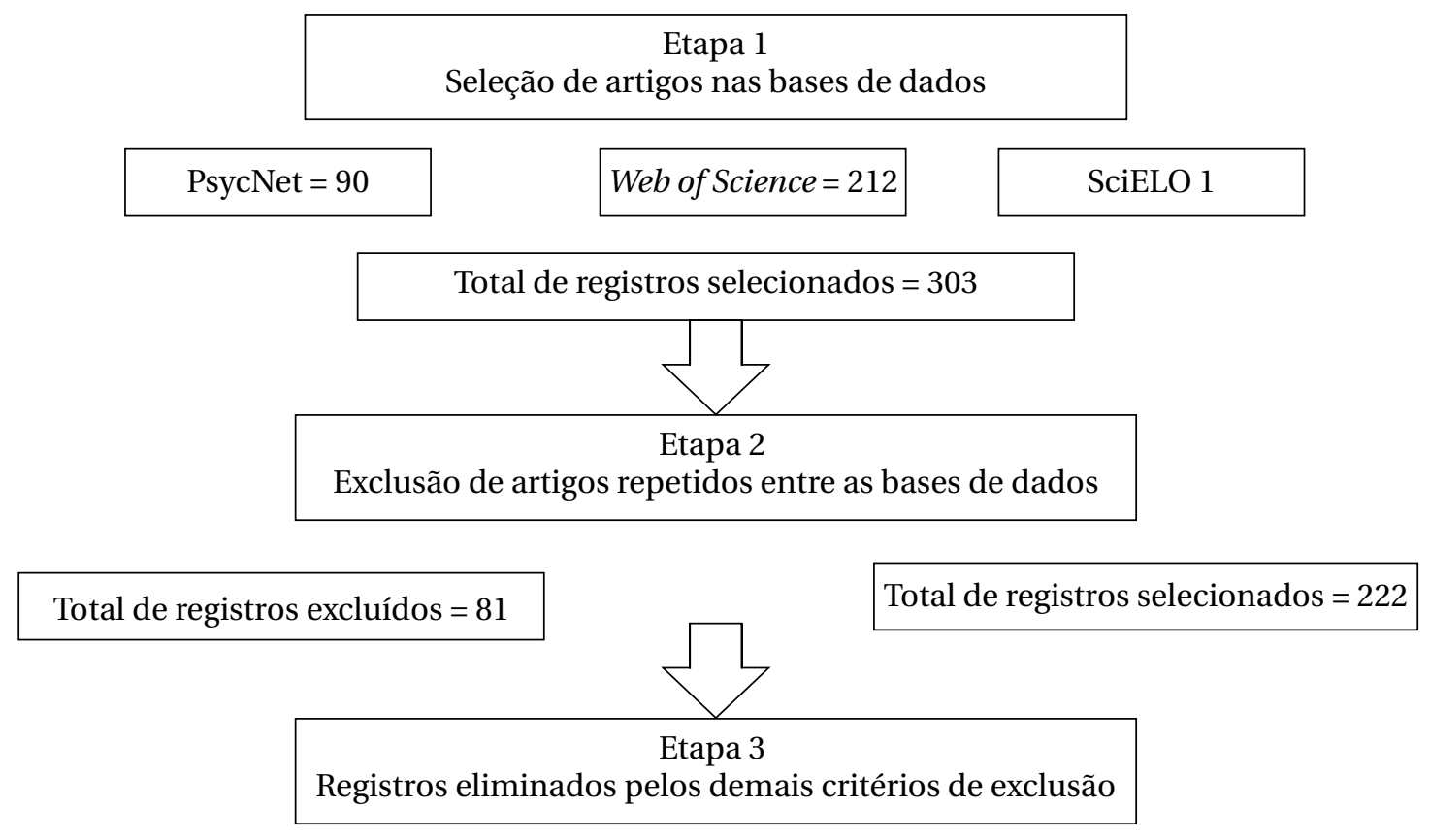

Total de registros excluídos = 185

Total de registros selecionados $=37$

Total de registros excluídos $=28$

Total de registros selecionados $=9$

Figura 1

Fluxograma das etapas de busca e seleção dos artigos analisados

(Austrália $=1$ ). Esse resultado indica uma importante lacuna teórica e social para pesquisas internacionais e principalmente nacionais em relação à transição e adaptação de atletas em novos ambientes de trabalho culturalmente diferentes.

O termo que vem sendo utilizado na literatura brasileira para definir este processo de transição é "Expatriado" (Brandão et al., 2013; Pereira et al., 2005). Este termo compreende situações de transição de indivíduos entre regiões de um mesmo país e entre países culturalmente diferentes, motivada pela prática de uma modalidade esportiva. Na literatura internacional, os termos utilizados nos estudos são: “Acculturation", "Migration" ou “Migratory Transition" (Agergaard, 2008; Agergaard, \& Ryba, 2014; Blodgett et al., 2014; Evans, \& Stead, 2012; Garrido, Olmos, Arjona, \& Pardo, 2012; Richardson et al., 2012;
Ryska, 2001; Weedon, 2011). Entende-se por Aculturação a interpretação da cultura pelo indivíduo, por meio de um conjunto de fenômenos provenientes do contato direto e contínuo de grupos de indivíduos representantes de culturas diferentes (Ferreira, 1997; Pereira et al., 2005). Este processo envolve um amplo conjunto de reações emocionais e psicológicas e combinações cíclicas das quais o sujeito lança mão para lidar com diferenças culturais (Andreason, 2003).

Realizando uma análise descritiva sobre os nove artigos, pode-se observar que o principal interesse dos estudos sobre a transição de atletas para jogarem em novas localidades é entender as experiências e desafios enfrentados pelos atletas que optam por se deslocar para outra região ou país visando a prática esportiva e ascensão na carreira (Agergaard, 2008; Agergaard, \& Ryba, 2014; Baines, 2013; Blodgett et al., 
2014; Evans, \& Stead, 2012; Richardson et al., 2012; Ryska, 2001; Schinke et al., 2013; Tibbert et al., 2015; Weedon, 2011). Um dos estudos (Agergaard, \& Ryba, 2014), além da compreensão da experiência da transição migratória, também objetivou propor uma estrutura interdisciplinar no clube que contextualiza esse fenômeno e visou a contribuir para desenvolvimento da carreira do atleta.

Interessante observar que a totalidade dos estudos analisados apresentou delineamento qualitativo. Foram realizadas entrevistas, questionários, grupos focais para acessar a experiência dos atletas quanto ao processo de transição e adaptação a um novo contexto esportivo, o que é extremamente valioso, mas também compromete a generalização dos dados. Schinke et al. (2013) sugerem que metodologias qualitativas devem continuar sendo realizadas para compreender a complexidade deste processo que mostra-se multifacetado, fluido e socialmente construído. No entanto, embora a profundidade na compreensão desse fenômeno através da pesquisa qualitativa, variáveis importantes associadas a esse processo como ansiedade (Tibbert et al., 2015) e estresse (Schinke et al., 2013) poderiam ter sido melhor exploradas nos estudos encontrados integrando, por exemplo, instrumentos específicos na coleta de dados das pesquisas analisadas. Este número expressivo ou preponderante de estudos qualitativos pode também ser explicado pela especificidade da população em questão. Em razão de suas características e difícil acesso, os grupos de sujeitos podem ficar reduzidos e os pesquisadores optarem por estudos de viés qualitativo.

Quanto ao esporte predominante nas pesquisas encontradas, observa-se que as modalidades esportivas coletivas foram as mais pesquisadas, como: Futebol (Agergaard, \& Ryba, 2014; Baines, 2013; Richardson et al., 2012; Tibbert et al., 2015; Weedon, 2011), Handeball (Agergaard, 2008) e Rugby (Evans, \& Stead, 2014; Schinke et al. (2013) desenvolveram sua pesquisa com mais de uma modalidade integrando coletivas e individuais (hóquei no gelo, Natação, Boxe, Nado Sincronizado, Basquetebol, Ciclismo, Badminton, Tae Kwon do, Track and Field, Esqui), enquanto Blodgett et al. (2014) investigaram atletas de modalidades individuais (Arco e Flecha e Boxe).

Constata-se que o futebol foi o esporte mais pesquisado. $\mathrm{O}$ interesse maior nessa modalidade pode estar vinculado ao fato do desenvolvimento de estratégias pela a Associação de Futebol para a profissionalização cada vez mais cedo dos atletas (atletas muito jovens) (Weedon, 2011). Sendo assim, para garantir que o investimento nesses jovens atletas seja positivo e produtivo, clubes de futebol e profissionais ligados a essa área preocupam-se pelo fato dos atletas precisarem deslocarem-se para diferentes partes do mundo para a prática do esporte, deixando a família e sua cultura em um momento do desenvolvimento humano de grande impacto para a formação do indivíduo (Agergaard, \& Ryba, 2008; Muuss, 1969; Richardson et al., 2012; Santos, Melo Neto, \& Koller, 2014; Smith, 2011; Weedon, 2011). Quanto ao impacto/efeitos do processo de transição e adaptação para a vida dos indivíduos abordados nas pesquisas, os conteúdos foram agrupados em unidades temáticas definidas a partir dos resultados dos artigos investigados.

\section{Ajuste à filosofia no novo clube}

As pesquisas apontaram que uma das frequentes preocupações dos participantes investigados e diretamente relacionada ao desempenho na modalidade esportiva é a diferença na forma de treinamento dos novos ambientes de trabalho. Variáveis como intensidade e frequência dos treinos e jogos (Weedon, 2011), assim como a necessidade de se ajustar a um jogo mais rápido e agressivo (Richardson et al., 2012) foram apontados como fatores que dificultaram a adaptação do atleta no início de sua transição e provocaram o retorno antecipado ao local de origem (Agergaard, \& Ryba, 2014). Embora as diferentes modalidades esportivas apresentem demandas específicas (intensidade, força, velocidade) e apresentem regras federadas (DaCosta, 2005), a forma como os atletas são instruídos e incentivados a jogar varia de acordo com o clube e com o treinador. Para tanto, é esperado que no início de sua transição para um novo ambiente de trabalho, o atleta necessite reconhecer o ambiente e obter um entendimento do novo modelo de performance, assim como das expectativas com relação ao seu desempenho.

Constata-se também que a filosofia do novo clube esportivo mostra-se bastante influente nas atitudes e crenças do novo atleta. O caminho para o sucesso parece ser o atleta internalizar a filosofia do clube esportivo, vivendo expectativas e normas estabelecidas pelos profissionais e outros atletas direta- 
mente envolvidos na sua rotina. O clima grupal que os técnicos com suas ações proporcionam nos treinamentos claramente influencia o comportamento do atleta, conforme identificaram Tibbert et al. (2015). Ou seja, estes autores observaram que os técnicos impõem ao atleta a ideia que o excesso de treinamentos é fundamental, que é necessário aceitar e conviver com a dor, assim como esperam que o atleta rejeite suas emoções, pois esta é a postura esperada para se aculturar e se adaptar no clube. Os atletas, muitas vezes, submetem-se às necessidades do novo clube, comprometendo suas crenças e emoções. Sacrificam, assim, sua própria identidade para assumir a identidade imposta pelo novo clube.

\section{Distanciamento do ambiente familiar}

A distância física da família destacou-se como uma situação de conflito para os participantes dos estudos analisados nessa revisão. Constata-se que a transição para um novo local de trabalho provoca inúmeras mudanças e dificuldades ao atleta. Observa-se uma intensa saudade da família, o medo de residir com estranhos, o aumento no uso do telefone celular, redes sociais e internet para o contato com familiares, somada à dificuldade de enfrentar a morte de pessoas íntimas (Agergaard, \& Ryba, 2014; Blodgett et al., 2014; Evans, \& Stead, 2014; Richardson et al., 2012; Weedon, 2011).

Observa-se que a família desempenha um importante suporte na adaptação e manutenção dos atletas ao novo local de trabalho e residência (Lee, \& Kartika, 2014). Mesmo com apoio de profissionais especializados e dos demais companheiros de trabalho (atletas), a família é apontada como o suporte mais importante e significativo para essa transição entre diferentes localidades (Blodgett et al., 2014; Richardson et al., 2012). No entanto, embora haja a saudade e falta da família, observou-se que existe um receio por parte de alguns atletas em ir visitar a família e perder situações importantes no seu clube e até mesmo ser substituído por outro atleta pelo treinador (Richardson et al., 2012). Além disso, a pesquisa de Richardson et al. (2012) também mostra que a atitude de visitar a família é percebida pelos participantes como uma fraqueza ou incapacidade de lidar com a pressão dentro do novo ambiente de trabalho.

Já no estudo de Agergaard (2008), as participantes da pesquisa definiram como prioridade para sua tran- sição a um novo clube, a mudança junto com a família ou o contrato com um clube próximo à sua cidade. Dessa forma, estas atletas poderiam administrar o lado profissional com as demandas pessoais, diminuindo o risco de declínio na performance esportiva e sofrimento/riscos na adaptação à nova realidade.

\section{Competência linguística}

A análise dos principais impactos/efeitos dos artigos evidencia a comunicação como significativamente influente na adaptação a uma nova cultura (Agergaard, \& Ryba, 2014); Baines, 2013; Evans, \& Stead, 2014; Richardson et al., 2012; Schinke et al., 2013; Weedon, 2011). Estar em uma nova cultura especialmente com uma língua diferente, mostrou-se um grande desafio para os participantes. A língua não só interfere nas relações interpessoais, como prejudica no entendimento e interpretação das instruções dos professores e treinadores, podendo ser motivo de desinteresse do técnico no novo atleta (Richardson et al., 2012; Weedon, 2011).

Observa-se também que a competência linguística influencia diretamente na habilidade para se adaptar e adotar as normas da cultura local, assim como estabelecer relações interpessoais. Sentimentos como medo de se aproximar das pessoais locais, assim como a frustração e desânimo ao tentar interagir e não ser compreendido, são aparentes nas pesquisas em que os participantes não dominam a língua local (Baines, 2013; Evans, \& Stead, 2014; Schinke et al., 2013; Weedon, 2011). Portanto, reconhecida como a principal barreira para uma efetiva adaptação entre culturas, a falta de habilidade na comunicação e na linguagem aparece como fator principal para o fracasso na adaptação em locais de línguas diferentes (Andreason, 2003; Baines, 2013).

$\mathrm{Na}$ análise dos artigos, um dos estudos revelou que a língua não só torna-se uma barreira para a adaptação a um novo contexto esportivo como é manipulada por dirigentes e pela mídia para proteger os interesses do clube e dos atletas (Baines, 2013). No contexto da globalização do esporte, os atletas estrangeiros necessitam utilizar traduções e interpretações para se comunicar. No entanto, essa estratégia por vezes é manipulada pelos clubes para assegurar os interesses financeiros, caráter do atletas, confirmar as regras institucionais, regulamentos e reputação nacional e internacional do clube. 


\section{“Choque" cultural}

A transição para uma nova cultura pode provocar inicialmente um "choque" cultural a partir de uma reação ao novo, ao imprevisível e às incertezas do ambiente (Andreason, 2003). O desconhecimento cultural e do ambiente físico e social, o sentimentos de solidão, a dificuldade de se adaptar às normas e as expectativas da nova cultura foram os principais problemas dos atletas apontados pelos estudos encontrados nessa busca (Agergaard, 2008; Agergaard, \& Ryba, 2014; Evans, \& Stead, 2012; Richardson et al., 2012; Weedon, 2011).

Os estudos de Blodgett et al. (2014), Evans e Stead (2014) e Richardson et al. (2012) apontam o isolamento social e da família como sentimento bastante presente no início de um processo de transição e fator de risco para a adaptação ao novo ambiente. $\mathrm{O}$ isolamento provoca uma sensação de rompimento com a cultura de origem e desafia o indivíduo no sentindo de identidade e pertencimento a uma cultura. Ocorre, assim, um conflito na vida do atleta em função desta brusca troca de realidade (Blodgett et al., 2014; Schinke et al., 2013; Tibbert et al., 2015). Além disso, constata-se que experiências de frustração na tentativa de adaptar-se ao novo ambiente, provocam no atleta um distanciamento ao ambiente esportivo e uma aproximação com o ambiente, cultura e valores originais (Tibbert et al., 2015). No entanto, com o intuito de minimizar esses sentimentos, Evans e Stead (2014) apontaram para a importância das relações sociais e de apoio no processo de adaptação. Destaca-se ainda que tais relações precisam ser reais (presenciais), pois dificilmente serão compensadas/substituídas pelas relações virtuais (Evans, \& Stead, 2014). Outra importante iniciativa é a de realizar atividades culturais, de lazer ou integração no novo local (por exemplo, ir à igreja) e resgatar atividades praticadas na cultura de origem (Agergaard, \& Ryba, 2014).

Na perspectiva da adaptação ao novo, o grau de instrução formal, assim como nível técnico, também influenciam. Na pesquisa de Evans e Stead (2014), as pessoas com adequado nível de escolaridade (médio e superior) e elevado nível técnico no esporte foram capazes de estabelecer mais saudáveis e mais positivas relações interpessoais e, consequentemente, se adaptarem melhor à nova realidade. Enquanto isso, atletas de grau de instrução inferior associado ao nível socioeconômico menos favorecido apresentaram dificuldades em construir relações e sentiram certo desinteresse por parte dos indivíduos locais em suas culturas, dificultando a aproximação/adaptação (Blodgett et al., 2014; Evans, \& Stead, 2014).

A identidade cultural não é anulada quando o sujeito sai de sua cultura de origem e vai para uma nova cultura (Cuadrado, \& Tabernero, 2014). Ao contrário, as diferentes culturas devem ser complementadas e usadas como recursos para negociar as experiências e comportamentos dos atletas no contexto esportivo de acolhimento (Schinke et al., 2013; Weedon, 2011). A pesquisa de Schinke et al. (2013) aponta que os atletas encaram dificuldades ligadas à adaptação sem o suporte dos colegas de equipe e dos técnicos no início. No entanto, quando o atleta considera e aceita o estresse diante da nova realidade, este tende a buscar ajuda junto aos demais colegas de equipes para seu processo de adaptação. A troca de experiências com outros atletas tende a facilitar o processo de adaptação (Schinke et al., 2013).

\section{Considerações finais}

Pode-se concluir que o estudo apresenta conhecimentos para o entendimento do fenômeno do expatriado no contexto esportivo. Conclui-se que o processo de expatriado é emergente e que deve ser estudado nos dias atuais, uma vez que identifica-se acentuada mobilidade entre culturas dos indivíduos e que este processo pode ser um fator de risco na formação destes atletas de alto nível do esporte.

A partir dos estudos foi possível constatar que a transição para uma nova cultura é um processo que exige acompanhamento e orientação. Observou-se a não existência de um único termo para definir esse processo de transição no ambiente esportivo. Transição migratória, migração e aculturação são os termos mais utilizados. Preocupados com esse fenômeno, o grande interesse dos pesquisadores dos estudos residiu em compreender como ocorre os atletas percebem este processo de transição e adaptação para uma nova cultura. Os desfechos mais frequentes nos estudos analisados foram dificuldades de adaptação relacionadas à língua - principalmente quando os treinadores falam muito rápido e quando são necessários intérpretes - distância da família e diferenças culturais (Agergaard, 2008; Agergaard, \& Ryba, 2014; Baines, 2013; Blodgett et al., 2014; Evans, \& Stead, 2014; Richardson et al., 2012; Ryska, 2001; Schinke et al., 2013; Tibbert et al., 2015; Weedon, 2011). Dessa forma, ainda que os atletas estejam motivados à 
projeção na carreira esportiva, os desafios decorrentes dessa trajetória, como adaptação ao novo estilo de treinamento e outras dificuldades podem levá-los a passar por situações de isolamento e dificuldades de estabelecer relações interpessoais (Agergaard, 2008; Agergaard, \& Ryba, 2014; Blodgett et al., 2014; Evans, \& Stead, 2014; Richardson et al., 2012; Ryska, 2001; Weedon, 2011). Os resultados dos estudos (Agergaard, 2008; Agergaard, \& Ryba, 2014; Baines, 2013; Blodgett et al., 2014; Evans, \& Stead, 2014; Richardson et al., 2012; Ryska, 2001; Schinke et al., 2013; Tibbert et al., 2015; Weedon, 2011) sugerem que, com a globalização do esporte e a crescente mobilidade dos atletas, os clubes e locais de trabalho de atletas precisam desenvolver estratégias para intervir e promover a saúde desses indivíduos expatriados.

Importante ressaltar também que as pesquisas aqui analisadas apresentavam um delineamento qualitativo, buscando através de entrevistas entender o fenômeno de transição entre culturas para a prática do esporte. Novas pesquisas são necessárias com o objetivo de investigar outras variáveis associadas ao sentimento de isolamento e dificuldades de estabelecer relações e que podem se intensificar em ambientes de muita pressão como o esporte. Dentre elas, sugere-se o estudo que foque o estresse, a ansiedade entre outros. Para tanto, estudos quantitativos e até mesmo de delineamentos mistos fazem-se neces-

\section{Referências}

Andreason, A. W. (2003). Expatriate adjustment to foreign assignments. International Journal of Commerce \& Management, 13(1), 42-60. doi:10.1108/eb047459

Agergaard, S. (2008). Elite athletes as migrants in Danish women's handball. International Review for the Sociology of Sport, 43(1), 5-19. doi:10.1177/1012690208093471

Agergaard, S., \& Ryba, T. V. (2014). Migration and career transition in professional sports: transnational athletic careers in a psychological and sociological perspective. Sociology of Sport Jornal, 31(2), 228-247. doi:10.1123/ssj.2013-0031

Baines, R. (2013). Translation, globalization and the elite migrant athlete. The Translator, 19(2), 207-228. doi:10.1080/13556509.2013.10799542

Blodgett, A. T., Schinke, R. J, McGannon, K. R., Caholic, D. A., Enosse, L., Peltier, D., \& Pheasant, C. (2014). sários e poderão contribuir ainda mais para o entendimento do fenômeno, aumentando a integração compreensiva e potencial de generalização dos dados.

Por fim, é necessário ponderar sobre algumas limitações deste estudo. A busca por artigos envolvendo o fenômeno do expatriado restringiu-se a três bases de dados além de utilizar alguns termos não indexados devido à especificidade do tema. Considera-se que nem todas as pesquisas com essa temática encontram-se em periódicos indexados nessas bases; sendo assim, estudos potencialmente relevantes podem não ter feito parte dessa amostra avaliada. Além disso, considera-se a possibilidade de existirem outros termos utilizados para abordar essa temática e que não foram utilizados nessa pesquisa, já que localidades distintas no mundo utilizam diferentes termos para tratar desse assunto. Contudo, embora a quantidade de artigos analisados tenha sido relativamente pequena, foi possível levantar algumas características do panorama dos impactos/ efeitos do processo de transição e adaptação de atletas no mundo, gerando pistas pra intervenção focal é preventiva e aumentando a compreensão sobre esta transição entre culturas. Estas evidências possibilitaram a discussão da emergência de novas pesquisas nessa área, especialmente em países subdesenvolvidos que também mostram-se grandes potências no esporte (por exemplo, Brasil e México).

Navigating the inseder-outsider hyphen: a qualitative exploration of the acculturation challenges of aboriginal athletes pursuing sport in Euro-Canadian context. Psychology of Sport and Exercise, 15(4), 345-355. doi:10.1016/j.psychsport.2014.02.009

Brandão, M.R.F., Magnani, A., Tega, E., \& Medina, J. P. (2013). Além da cultura nacional: o expatriado no futebol. Revista Brasileira Ciência e Movimento, 21(2), 177182. doi:10.18511/0103-1716/rbcm.v21n2p177-182

Carpes, A., Scherer, F, Diniz, D., \& Beuron, T. A. (2011). Expansão internacional para a China e seu reflexo no ajustamento intercultural do indivíduo brasileiro expatriado. Revista Ibero-Americana de Estratégia, 10(3), 22-48. Recuperado de http://www.revistaiberoamericana.org/ojs/index.php/ibero/article/view/1768/pdf

Cuadrado, E., \& Tabernero, C. (2014). Dispositional and psychosocial variables as longitudinal predictors of acculturative stress. Applied Psychology, 63(3), 441-479. doi:10.1111/j.1464-0597.2012.00531.x 
DaCosta, L. (Org.). (2005). Atlas do esporte no Brasil: atlas do esporte, educação física e atividades físicas de saúde e lazer no Brasil. Rio de Janeiro, RJ: Shape.

Evans, A. B., \& Stead, D. E. (2014). "It's long way to the super league": the experiences of Australisian professional rugby league migrants in the United Kingdon. International Review for the Sociology of Sport, 49, 707-727. doi:10.1177/1012690212464700

Ferreira, A. B. H. (1997). Minidicionário Aurélio de língua portuguesa. Rio de Janeiro, RJ: Nova Fronteira.

Garrido, A. A., Olmos, J. C. C., Arjona, N. G., Pardo, R. (2012). Immigration, school, physical activity and sport: analysis of sport acculturation in Spain. Kinesiology, 44(1), 83-93. Recuperado de http:// hrcak.srce.hr/index.php?show=clanak\&id_clanak_ jezik $=124407$

Kubo, E. K. M., \& Braga, B. M. (2013). Ajustamento intercultural de executivos japoneses expatriados no Brasil: um estudo empírico. Revista de Administração de Empresas, 53(3), 243-255. doi:10.1590/S0034-75902013000300003

Lee, L., \& Kartika, N. (2014). The influence of individual, family, and social capital factors on expatriate adjustment and performance: the moderating effect of psychology contract and organizational support. Expert Systems with Applications, 41(11), 5483-5494. doi:10.1016/j.eswa.2014.02.030

Moher, D., Liberati, A., Tetzlaff, J., Altman, D.G. (2009). Preferred reporting items for systematic reviews and meta-analyses: The PRISMA statement. PLoS Med, 6(7), e1000097. doi:10.1371/journal.pmed.1000097

Muuss, R. E. (1969). Teorias da adolescência. Belo Horizonte, MG: Editora do Professor.

Pereira, N. A. F., Pimentel, R., \& Kato, H. T. (2005). Expatriação e estratégia internacional: o papel da família como fator de equilíbrio na adaptação do expatriado. Revista de Administração Contemporânea, 9(4), 53-71. doi:10.1590/S1415-65552005000400004

Reis, F. A. (2015). Gestão de expatriados no Brasil: a percepção de expatriados japoneses à luz da teoria do ajustamento cultural. Revista Foco, 8(1), 128-141. Recuperado de http://www.novomilenio.br/periodicos/index.php/foco/article/view/142/ 104
Rial, C. (2008). Rodar: a circulação dos jogadores de futebol brasileiros no exterior. $\mathrm{Ho}$ rizontes Antropológicos, 14(30), 21-75. doi:10.1590/S0104-71832008000200002

Richardson, D., Littlewood, M., Nesti, M., \& Benstead, L. (2012). An examination of the migratory transition of elite young European soccer players to the english premier league. Journal of Sport Sciences, 30(15), 1605-1618. doi:10.1080/02640414.2012.733017

Rosal, A. S. R. (2015). Gestão de recursos humanos internacional e o ajustamento intercultural do executivo expatriado. Psicologia Revista. Revista da Faculdade de Ciências Humanas e da Saúde, 24(1), 121-141. Recuperado de http://revistas.pucsp.br/ index.php/psicorevista/article/view/24232/17442

Ryska, T. A. (2001). The impact of acculturation on sport motivation among Mexican-American adolescent athletes. The Psychological Record, 51(4). 533-547. Recuperado de http://opensiuc.lib. siu.edu/tpr/vol51/iss $4 / 2$

Santos, E. C., Melo Neto, O. C., \& Koller, S. H. (2014). Adolescentes e adolescências. In L. F. Habigzang, E.Diniz, \& S. H Koller (Eds.), Trabalhando com adolescentes: teoria e intervenção psicológica (pp 18-29). Porto Alegre, RS: Artmed.

Schinke, R. J., McGannon, K. R., Battochio, R. C., \& Wells, G. D. (2013). Acculturation in elite sport: a thematic analysis of immigrant athletes and coaches. Journal of Sports Sciences, 31(15), 1676-1686. doi:10.1080/02640414.2013.794949

Smith, P. K. (2011). Understanding children's development (5th ed.). Chichester: Wiley.

Tibbert, S. J., Andersen, M. B., \& Morris, T. (2015). What a difference a "Mentally Toughening" year makes: the acculturation of a rookie. Psychology of Sport and Exercise, 17, 68-78. doi:10.1016/j.psychsport.2014.10.007

Urrútia, G., \& Bonfill, X. (2010). La declaración PRISMA: una propuestapara mejorar la publicación de revisiones sistemáticas y metanálisis. Medicina Clínica (Barcelona), 135(11), 507-511. doi:10.1016/j.medcli.2010.01.015

Weedon, G. (2011). 'Glocal boys': exploring experiences of acculturation amongst migrant youth footballers in premier league academies. International Review for the Sociology of Sport, 47(2), 200-216. doi:10.1177/1012690211399221 


\section{Fernanda Faggiani}

Mestre em Psicologia do Esporte, Programa de PósGraduação em Psicologia da Pontifícia Universidade Católica do Rio Grande do Sul (PUCRS)

\section{Daniele Lindern}

Mestre em Psicologia, Programa de Pós-Graduação em Psicologia da Pontifícia Universidade Católica do Rio Grande do Sul

\section{Artur Strey}

Graduando em Psicologia e bolsista de iniciação científica da PUCRS.

Paula Fernandes Aiquel

Graduanda em Psicologia e auxiliar de pesquisa da PUCRS.

\section{Daniel Fulginiti}

Graduando em Psicologia e bolsista de iniciação científica da PUCRS.

\section{Camila Sartori}

Graduanda em Psicologia e bolsista de iniciação científica da PUCRS.

\section{Carolina Saraiva de Macedo Lisboa}

Doutora em Psicologia, Programa de Pós-Graduação em Psicologia da Pontifícia Universidade Católica do Rio Grande do Sul.

Endereço para envio de correspondência: Programa de Pós-Graduação em Psicologia Faculdade de Psicologia Pontifícia Universidade Católica do Rio Grande do Sul Av. Ipiranga, 6681 - Prédio 11, sala 929

Porto Alegre - RS - BRASIL

CEP 90619-900

Fone/Fax + 55513320.3500 ramal 7750 “

Recebido 02/06/2016

Aprovado 12/08/2016

Received 06/02/2016

Approved 08/12/2016

Recibido 02/06/2016

Aceptado 12/08/2016

Como citar: Faggiani, F, Lindern, D., Strey, A.; Aiquel, P. F, Fulginiti, D., Sartori, C., Lisboa, C. S. M.

(2016). O fenômeno do expatriado no contexto esportivo. Psicologia: Ciência e Profissão, 36(3): 738-747.

doi: 10.1590/1982-3703001832016

How to cite: Faggiani, F., Lindern, D., Strey, A.; Aiquel, P. F., Fulginiti, D., Sartori, C., Lisboa, C. S. M. (2016). The phenomenon of expatriates in the sports context. Psicologia: Ciência e Profissão, 36(3): 738-747. doi: 10.1590/1982-3703001832016

Cómo citar: Faggiani, F., Lindern, D., Strey, A.; Aiquel, P. F., Fulginiti, D., Sartori, C., Lisboa, C. S. M. (2016). El fenómeno de los expatriados en el contexto deportivo. Psicologia: Ciência e Profissão, 36(3): 738-747. doi: 10.1590/1982-3703001832016 